\title{
A novel differential diagnosis for diffuse cavitating nodules: primary pulmonary plasmacytoma
}

\author{
Shruthi Panduranga ${ }^{{ }^{*}}\left(\mathbb{0}\right.$, Samson Kade ${ }^{1}$, Swarna Shivakumar ${ }^{2}$, Harisha $V^{1}$ and Ravindra M. Mehta ${ }^{3}$
}

\begin{abstract}
Background: Extramedullary plasmacytoma is a rare monoclonal plasma cell neoplasm that originates from tissues other than the bone marrow and constitutes only 3\%-5\% of all plasma cell neoplasms. Most cases involve the upper respiratory tract. Extramedullary plasmacytoma involving the lung is extremely rare. Primary pulmonary plasmacytoma is a rare type of extramedullary plasmacytoma, usually presenting with a nodule or mass in hilar region. Literature search has shown very few cases of immunohistochemically confirmed cases of pulmonary plasmacytoma. Diffuse pulmonary infiltration is an unusual presentation.

Case presentation: We report the case of a 56 year old lady with history of cough and breathlessness since one month. Computed Tomography revealed diffusely scattered multiple cavitating nodules and consolidation in both lungs. Computed Tomography guided biopsy of one of the lung nodules was done. Histopathologic examination and immunohistochemistry showed features of pulmonary plasmacytoma. This is an unique case of primary pulmonary plasmacytoma with the rare presentation as diffusely scattered multiple cavitating nodules and consolidation. According to our literature search, primary pulmonary plasmacytoma manifesting as cavitating nodules is being reported for the first time.
\end{abstract}

Conclusions: Primary pulmonary plasmacytoma should be also be considered in the differential diagnosis of multiple diffusely scattered cavitating lung nodules.

Keywords: Pulmonary neoplasms, Plasmacytoma, Cavitating nodules, Diffuse lung nodules, Case report

\section{Background}

Extramedullary plasmacytoma (EMP) is a monoclonal plasma cell tumour that originates from tissues other than the bone marrow [1]. Most cases (80-90\%) involve the nasal and paranasal cavities, nasopharynx, larynx, and upper aerodigestive tract $[1,2]$. EMP involving the lung is extremely rare $[3,4]$.

Primary pulmonary plasmacytoma (PPP) usually presents with a nodule or mass in hilar region [1-4]. Here we report a case of PPP presenting as diffusely distributed

\footnotetext{
*Correspondence: drshruthirithin@gmail.com

1 Department of Radiology and Imaging, Apollo Speciality Hospital,

Jayanagar, Bangalore 560011, India

Full list of author information is available at the end of the article
}

multiple cavitating nodules and consolidation, which is exceedingly rare. The present case is the first report of PPP presenting as diffusely distributed multiple cavitating nodules in both lungs.

\section{Case presentation}

A 56 year old lady, non-smoker, without a familial lung cancer history, presented to the respiratory clinic complaining of cough, breathing difficulty since one month. Past medical history, family and psycho-social history were unremarkable. Physical examination showed no abnormalities. A routine laboratory work-up showed increased C- reactive protein with normal blood counts, biochemistry, transaminase level, erythrocyte 
sedimentation rate and protein studies. Bence Jones protein test in urine was negative after 24 hours.

The chest radiograph showed multifocal diffuse confluent nodular opacities in both lungs and consolidation in bilateral lower zones. High resolution computed tomography scan (HRCT) revealed multiple discrete and confluent solid as well as ground glass nodules diffusely scattered in both lungs, predominantly in lower lobes .Most of the nodules showed central cavitation. (Fig 1a, b, c, d and Fig 2) Patchy consolidation were seen in right middle and bilateral lower lobes (Fig 1c, d and Fig 2 ). Mild right pleural effusion was noted. No obvious enlarged lymph nodes were found in the mediastinum. No lytic lesions were seen in spine and bony thoracic cage. Visualised both breast parenchyma appear normal. Based on imaging findings, metastases and granulomatous disease were kept as differential diagnosis.

CT guided core needle biopsy of one of the lung nodules was done under strict aseptic precautions.

Microscopy on histopathology (Fig 3a) showed features suggestive of small round cell tumor with differential diagnosis as plasmacytoma and neuroendocrine tumor. Immunohistochemistry (Fig $3 \mathrm{~b}$ ) revealed neoplastic plasma cells showing strong diffuse expression of CD138, CD45, vimentin and kappa, focally positive

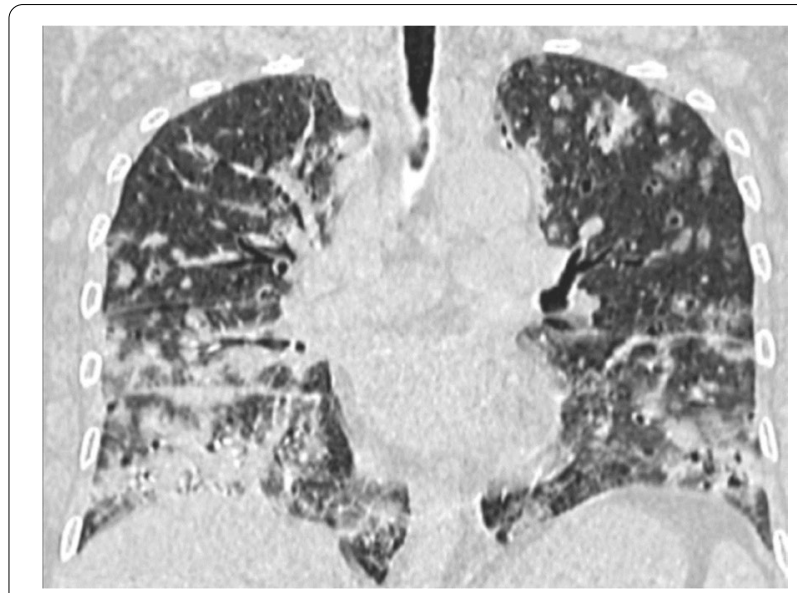

Fig. $2 \mathrm{HRCT}$ chest coronal section shows diffuse multiple cavitating nodules with consolidation in both lower lobes

for lambda with kappa:lambda ratio of 8:1. Features were suggestive of plasmacytoma. Bone marrow aspiration showed few plasma cells $(<5 \%)$ with normal skeletal survey. Hence proved as PPP. The patient received chemotherapy for more than 1.5 years and remains in good general condition.
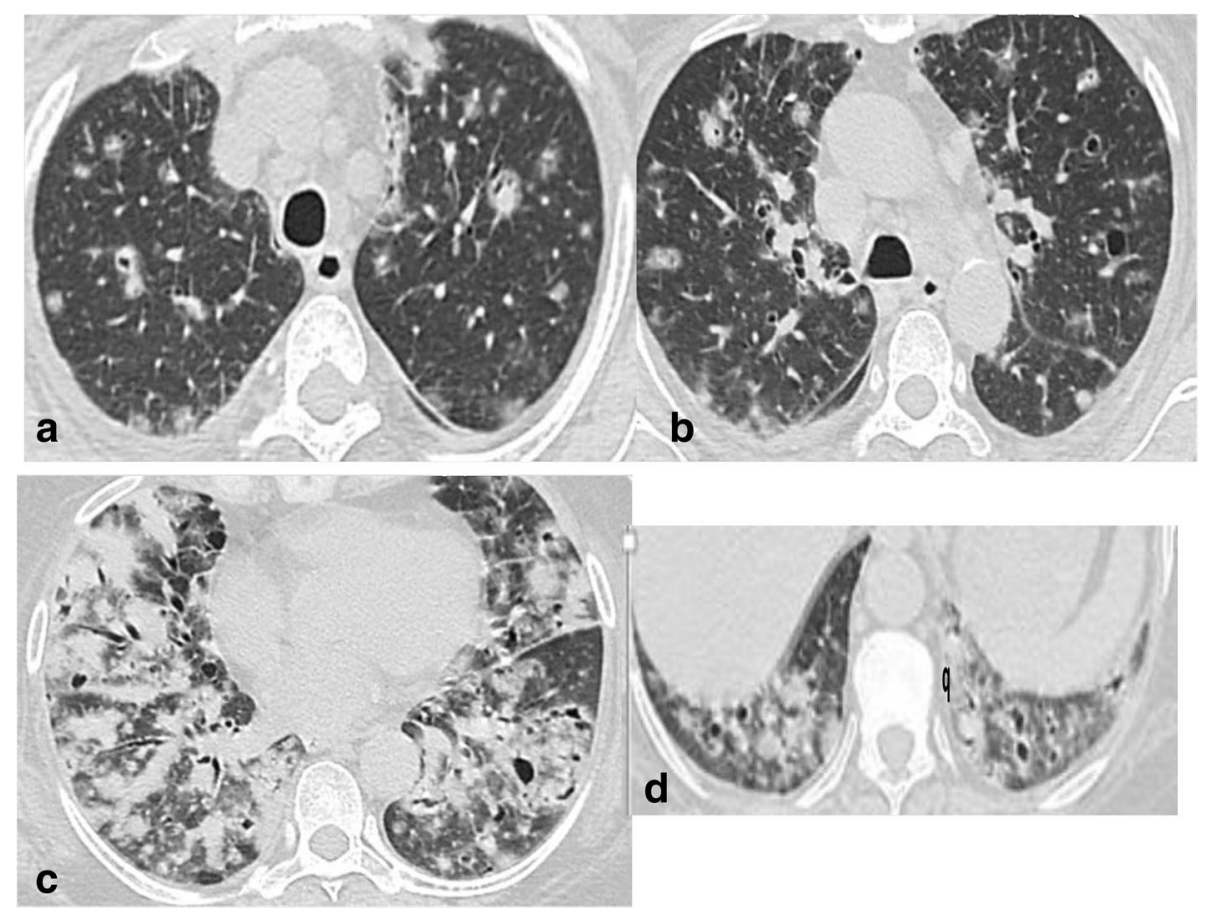

Fig. 1 HRCT chest axial sections show $\mathbf{a}$, b Diffusely scattered multiple cavitating nodules in both lungs $\mathbf{c}$, $\mathbf{d}$ confluent nodules and consolidation in both lower lobes 

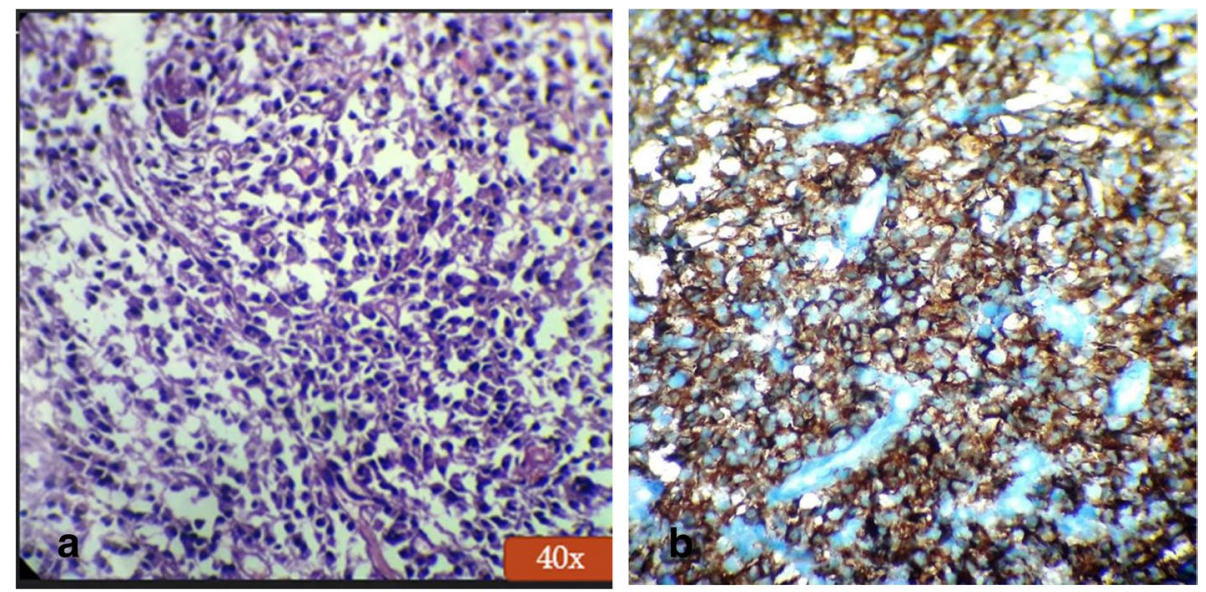

Fig. 3 a Histopathological examination showing diffuse sheets of round to plasmacytoid cells $\mathbf{b}$ Immunohistochemistry showing CD 138+

\section{Discussion}

Plasmacytoma can be classified into: multiple myeloma $(\mathrm{MM})$, isolated plasmacytoma, extramedullary plasmacytoma (EMP) and plasmablastic sarcoma [5].

MM is a systemic condition with monoclonal proliferation of plasma cells primarily involving the bone marrow. EMP is malignant proliferation of single clone of plasma cells arising outside the bone marrow in soft tissues or an organ and may infiltrate adjacent lymph nodes or cause distant metastasis[1]. EMP commonly arises as a solitary tumor in the upper respiratory tract mostly involving the nasal, oral cavity, paranasal sinuses and nasopharynx [1, 2]. Rarely, EMP arises in the lungs [3, 4]. PPP in the lung is extremely rare, although pulmonary involvement with multiple myeloma is more common. Literature review reveals few cases of PPP and is mostly localised to the hilar region as a solitary nodule or mass $[1,3,4]$. Here we report an unusual case of PPP presenting as diffusely distributed multiple cavitating nodules and consolidation.

In the upper airway, submucosa is the most common location for plasmacytoma due to abundance of plasma cells and continuous antigenic exposure [1]. In the lower airway, plasmacytoma occurs in the tracheobronchial tree, hilum [3] or, rarely, in the lung parenchyma [6].

PPP has no particular sex predilection with a median age of 55 years [3, 4, 7]. Serum M protein or Bence-Jones light chains in urine are negative in EMP [7]. Patients are mostly asymptomatic. Joseph et al reported that that nearly half of the cases are asymptomatic with the rest of them showing non-specific symptoms such as cough, dyspnea, chest pain and fever [3, 4]. Clinically PPP is divided into three stages as follows: stage I: the tumor is confined to the primary site; stage II: the tumor has invaded local lymph nodes; stage III: there are obvious widespread metastases [8]. Our case should be classified as stage I based on this. The radiographic presentation is that of a solitary mass or nodule mostly in hilar areas [4]. Typical HRCT manifestation of PPP are solitary lung nodules or masses localised to the hilar region or the lung parenchyma with a tendency for lower lobes. Nodules or masses are round to ovoid in shape, homogenous in appearance with smooth, well delineated margins and sizes ranging from 1.5-6 cm. Few cases have been reported with the rare presentation of diffuse alveolar consolidation [6,9-11] and it suggests that the tumor cells infiltrate into the lung parenchyma $[3,6,10]$.

Horiuchi et al. [6] reported a case of 45-year-old woman with fever and hypoxemia showing bilateral reticulonodular infiltration in lower lobes. Another case was reported by Niitsu et al. [10] of bilateral lung involvement and marked hypergammaglobulinemia in a 71-year-old asymptomatic woman. Lazarevic et al. [9] also reported a case with diffuse bilateral lung opacities in an elderly male.

In the current case, the presentation was unusual with diffusely scattered multiple cavitating nodules and consolidation which was initially misdiagnosed as granulomatous disease or metastases. These findings suggest PPP should be considered in the differential diagnosis of multiple cavitating lung nodules. PPP presenting as single or multiple nodules in the lung is often misdiagnosed as organizing pneumonia, tuberculosis, or lung cancer.

The diagnosis of PPP mainly depends on HPE, while CD138 and CD38 are characteristically positive in plasmacytoma. The pathologic differential diagnoses includes MALT lymphoma with plasma cell differentiation [7].

Differentiation of EMP from MM is based on plasma cell infiltration in lung lesions on HPE for a diagnosis of PPP. Normal bone marrow (lower than $5 \%$ plasma cells with no dyscrasia), normal skeletal survey, no related 
organ or tissue impairment, and no serum and/or urine $M$ protein has to be confirmed to fulfill the definitive diagnostic criteria for PPP which was true in our case [7]. PPP can potentially progress to multiple myeloma. Because of limited literature and its rarity, management and therapeutic options are variable. Both surgery and radiotherapy are good options because of its presentation as a single pulmonary mass. However, when the pulmonary involvement is diffuse, chemotherapy is best choice [7]. Previous cases with diffuse involvement have been treated with melphalan and prednisolone [6]. Surgical resection can be considered curative if it is complete and follow up is advised. Koss et al reported that overall 2 -year and 5-year survival rates were $66 \%$ and $40 \%$ [4].

\section{Conclusions}

PPP is a rare type of EMP with difficulty in diagnosis because of its non-specific imaging features. It usually presents as a nodule or mass in the hilar region. PPP presenting as multiple cavitating nodules and consolidation in both lungs is extremely rare; to our knowledge, no cases have been reported in the literature. We report this case to be aware of this unusual finding which can aid in reducing the incidence of errors and missed diagnosis.

\section{Abbreviations}

PPP: Primary pulmonary plasmacytoma; EMP: Extramedullary plasmacytoma; HPE: Histopathological examination; MM: Multiple Myeloma; HRCT: High resolution Computed Tomography; CT: Computed Tomography.

\section{Acknowledgements}

Not applicable

\section{Authors' contributions}

All authors had substantial contributions to the conception of the case report. All authors were active participants in the drafting and revising of the case report. All authors approved the final version of manuscript. All authors agree to be accountable for all aspects of the work. All authors were directly involved in this patient's care and contributed to the writing and editing of this manuscript. SK, RM researched and wrote the patient background and pulmonary history. HV, SS researched and wrote the abstract and conclusion of the manuscript, captured and arranged the images. SP conceptualized the process; researched, compiled, and wrote the procedure, conclusion, and introduction; and served as the overall editor.

\section{Funding}

Nil.

\section{Availability of data and materials}

Provided in this case report.

\section{Declarations}

Ethics approval and consent to participate

Ethics has approved this study.

\section{Consent for publication}

The authors certify that they have obtained all appropriate patient consent forms. In the form the patient has given her written informed consent for her images and other clinical information to be reported in the journal. The patients understand that their names and initials will not be published and due efforts will be made to conceal their identity, but anonymity cannot be guaranteed.

\section{Competing interests}

The authors declare that they have no competing interests.

\section{Author details}

${ }^{1}$ Department of Radiology and Imaging, Apollo Speciality Hospital, Jayanagar, Bangalore 560011, India. Department of Pathology, Apollo Hospital, Banerghatta Road, Bangalore 560011, India. ${ }^{3}$ Department of Pulmonology and Critical Care, Apollo Speciality Hospital, Jayanagar, Bangalore 560011, India.

Received: 2 Auqust 2021 Accepted: 14 November 2021

Published online: 07 December 2021

\section{References}

1. Wiltshaw E (1976) The natural history of extramedullary plasmacytoma and its relation to solitary myeloma of bone and myelomatosis. Medicine (Baltimore) 55:217-238

2. Dimopoulos MA, Hamilos G (1976) Solitary bone plasmacytoma and extramedullary plasmacytoma. Curr treat options Oncol. 2002; 3(3):255- 9. 2. Wiltshaw $E$. the natural history of extramedullary plasmacytoma and its relation to solitary myeloma of bone and myelomatosis. Medicine (Baltimore) 55(3):217-238

3. Joseph G, Pandit M, Korfhage L (1993) Primary pulmonary plasmacytoma. Cancer 71:721-724

4. Koss MN, Hochholzer L, Moran CA, Frizzera G (1998) Pulmonary plasmacytomas: a clinicopathologic and immunohistochemical study of five cases. Ann Diagn Pathol 2(1):1-11

5. Fanning SR, Hussain MA, Perez-Zincer F. "Plasmacytoma, extramedullary." E medicine. 2006. https://www.emedicine.com/med/topic2532.htm. Accessed 1 Dec 2021

6. Horiuchi T, Hirokawa M, Oyama Y et al (1998) Diffuse pulmonary infiltrates as a roentgenographic manifestation of primary pulmonary plasmacytoma. Am J Med 105(1):72-74

7. Taheri ZM, Mohammadi F, Karbasi M, Seyfollahi L, Kahkoei S, Ghadiany M, Fayazi N, Mansouri D (2010) Primary pulmonary Plasmacytoma with diffuse alveolar consolidation: a case report. Pathol Res Int Article ID 463465:3. https://doi.org/10.4061/2010/463465

8. Nie S, Peng DC, Gong HH, Ye CL, Nie X, Li HJ et al (2016) Primary pulmonary plasmacytoma: a case report introduction. World J Surg Oncol 14:205. https://doi.org/10.1186/s12957-016-0948-8

9. Lazarevic V, Cemerikic-Martinovic V, Suvajdzic N, Subotic D, Colovic M (2001) Diffuse primary plasmacytoma of the lung. Haematologia (Budap) 31:161-165

10. Niitsu N, Kohri M, Hayama M et al (2005) Primary pulmonary plasmacytoma involving bilateral lungs and marked hypergammaglobulinemia:dif ferentiation from extranodal marginal zone B-cell lymphoma of mucosaassociated lymphoid tissue. Leuk Res 29(11):1361-1364

11. Kim S-H, Kim TH, Sohn JW, Yoon HJ, Shin DH, Kim IS, Par SS (2012) Primary pulmonary Plasmacytoma presenting as multiple lung nodules. Korean J Intern Med 27:111-113. https://doi.org/10.3904/kjim.2012.27.1.111

\section{Publisher's Note}

Springer Nature remains neutral with regard to jurisdictional claims in published maps and institutional affiliations. 